\title{
Effect of Atmospheric Pressure Plasma Modification on Polyimide and Adhesive Joining with Titanium
}

\author{
M. AKRAM, K.M.B. JANSEN, L.J. ERNST, S. BHOWMIK, G. AJEESH, S. AHMED, and \\ D. CHAKRABORTY
}

\begin{abstract}
This investigation highlights the effect of surface modification on polyimide by atmospheric pressure plasma treatment with different exposure time. Surface modification of polymer by plasma treatment essentially creates physical and chemical changes such as cross-linking and formation of free radicals. It also forms oxygen functionalization in the form of polar groups on polymer surface, hence improving the wetting and adhesion properties. It is observed that surface energy of the polymer increases with increasing exposure time of atmospheric pressure plasma. However, prolonged exposure time of plasma results in deterioration of the surface layer of polyimide resulting in degradation and embrittlement. Scanning electron microscopy and atomic force microscopy analysis reveal that there is a considerable morphological change on the polymer surface due to atmospheric pressure plasma treatment. X-ray photo electron spectroscopy analysis reveals that the oxygen functionalities of polymer surface increases significantly when polyimide is exposed to atmospheric pressure plasma. Untreated and atmospheric pressure plasma-treated polyimide sheet are adhesive bonded by employing polyimide adhesive as well as with titanium substrate. Due to surface modification of polyimide, it is observed that there is a significant increase in lap shear tensile strength, and therefore, this technology is highly acceptable for aviation and space applications.
\end{abstract}

DOI: $10.1007 / \mathrm{s} 11661-015-3067-1$

(C) The Minerals, Metals \& Materials Society and ASM International 2015

\section{INTRODUCTION}

IN recent times, considerable efforts are being made throughout the world for the development of polymers and composite materials, lighter in weight as well as with superior thermo-mechanical properties. These materials are often fabricated by adhesive bonding to form structural components. Adhesive bonding is replacing conventional joining methods such as riveting, welding, and mechanical fastening in a variety of applications because of better fatigue performance and high strengthto-weight ratio. ${ }^{[1,2]}$ Mechanical joining results in stress concentration and reduces the overall load carrying capacity of the structure. ${ }^{[3]}$ An adhesive joint can distribute the applied load over the entire bonded area with more uniform stress distribution and is suitable for joining dissimilar materials with low manufacturing cost. ${ }^{[3,4]}$ However, use of adhesive bonding for space application was restricted due to low thermal and mechanical properties of adhesives. Therefore, considerable effort is given to developing high-performance

M. AKRAM, K.M.B. JANSEN, and L.J. ERNST, are with the PME Department, Faculty of Mechanical, Maritime and Materials Engineering, Delft University of Technology, The Netherlands. Contact e-mails: s.bhowmik@tudelft.nl, b_shantanu@cb.amrita.edu S. BHOWMIK and G. AJEESH, are with the Department of Aerospace Engineering, Amrita University, Coimbatore, India. S. AHMED and D. CHAKRABORTY, are with the Department of Polymer Science and Technology, University of Calcutta, 92, APC Road, Kolkata 700009, India.

Manuscript Submitted, August 1, 2014.

Article published online July 21, 2015 adhesives for its essential application to space, and consequently, its application is increased rapidly in the last decades. ${ }^{[5,6]}$

In this context, this investigation highlights performance of recently developed ultrahigh temperatureresistant polyimide adhesive as service temperature of polyimide adhesive ranges from $223 \mathrm{~K}$ to $598 \mathrm{~K}$ $\left(-50{ }^{\circ} \mathrm{C}\right.$ to $\left.+375{ }^{\circ} \mathrm{C}\right)$. Polyimide adhesive also shows excellent resistance to acids, alkalis, solvents, corrosive agents, radiation, etc. and consequently extremely useful for aerospace and space applications. ${ }^{[7]}$

High temperature-resistant polymers such as polyimide show excellent physicochemical properties, and up to certain extent easy to process. However, very often they do not possess the desired surface properties which are essential for adhesive bonding. Polymers inherently show low surface energy than metals resulting in poor adhesion characteristics. Polymers are hydrophobic in nature and in general exhibit insufficient adhesive bond strength due to the relatively low surface energy. ${ }^{[8]}$ Therefore, surface properties like hydrophilicity and roughness on polymer surface is highly desirable for successful adhesive bonding. ${ }^{[9]}$ Due to these reasons, surface preparation of polymeric adherence is the most important factor for fabrication of polymer by adhesive bonding. The main purpose of surface preparation is to improve the adhesion properties to such an extent that adhesive failure does not take place. Surface treatment of polymers only impacts the region near to the surface and does not alter the bulk properties of the polymer.

A number of chemical and physical surface treatments have been developed for polymeric materials in recent 
years. Chemical modification techniques usually require wet or chemical reactions with polymer surface and that essentially alters the surface characteristics of the polymer. Physical surface modification includes corona discharge, ion or electron beam, photon beam, plasma discharge, and flame oxidation. Due to the disadvantage of chemical treatments, physical surface treatments are preferred to modify the surface of polymeric materials. ${ }^{[10]}$ Therefore, plasma surface treatment is often the preferred way to treat polymer surfaces and it offers more stable surface properties in comparison to any other treatments. However, conventional plasma treatment also has shortcomings. It requires a low pressure (partial vacuum) and thus, the parts must be processed in a vacuum chamber, restricting the part size. Atmospheric pressure plasma has been developed to operate at near ambient temperature and atmospheric pressure eliminating the expensive vacuum systems. ${ }^{[10,11]}$

Therefore, in the context, atmospheric plasma treatment was employed to modify the polymer surfaces. Egitto and Matienzo indicated that the plasmas can be used to modify polymer surfaces for adhesion enhancement by roughening the polymer surfaces, and/or introducing reactive chemical groups. ${ }^{[12]}$ The introduction of reactive chemical groups on polymer surfaces essentially improves wetting characteristics of polymer, resulting in spreading of coatings on polymer surface for better bonding. ${ }^{[13-15]}$

It has been emphasized that adhesion characteristic of polyimide to polyimide and polyimide to Titanium is a function of surface energy of polyimide film and related to the chemical compositions and chemical bonds of polyimide film. Therefore, the influence of chemical bonds such as $\mathrm{C}-\mathrm{O}, \mathrm{C}=\mathrm{O}$, and $\mathrm{C}-\mathrm{N}$ for the enhancement of adhesion performance of adhesive to polyimide sheet by air plasmas has been critically analyzed.

Surface morphology of the plasma-modified polyimide sheet was studied under Atomic Force Microscopy (AFM) and Scanning Electron Microscopy (SEM), and chemical functionalities of the polymer were studied under X-ray Photoelectron Spectroscopy (XPS). The improvement in adhesion properties of these materials after plasma treatment was correlated with lap shear strength of the Polyimide adhesive joints. Lap shear testing was carried out for bonded joints of all types of polymers in order to determine the joint strength.

\section{EXPERIMENTAL}

\section{A. Materials}

In this investigation, high temperature, thermally conductive polyimide adhesive 124-41, supplied by Creative Materials Inc, MA, USA, was used. Service temperature of this polyimide adhesive ranges from $218 \mathrm{~K}$ to $523 \mathrm{~K}\left(-55^{\circ} \mathrm{C}\right.$ to $\left.+250{ }^{\circ} \mathrm{C}\right)$ and exhibits tensile shear strength of $18 \mathrm{MPa}$. Before application of the adhesive to the substrate material, it was maintained under vacuum for 20 minutes at room temperature to remove any air bubbles.
Two test liquids, deionized water and formamide of known polar " $\gamma P$," and dispersion components " $\gamma D$ " of the surface energy were used to determine surface energies of the substrate materials through measurement of contact angle by the sessile drop method. The known components of the surface energy of liquids are listed in Table I.

Polyimide sheet MELDIN 7001 series supplied by Saint-Gobain Performance Plastics, Bristol USA was used in this study. This polyimide sheet shows tensile strength of $72.5 \mathrm{MPa}$ at $295.5 \mathrm{~K}\left(22.5^{\circ} \mathrm{C}\right)$ and $38 \mathrm{MPa}$ at $533 \mathrm{~K}\left(260{ }^{\circ} \mathrm{C}\right)$ and 8 pct elongation at room temperature. Polyimide sheets are thermally stable and also show good fire retardant and, consequently, widely used in high-temperature applications.

Titanium Grade 5 (TiA6V4) is one of the most widely used commercial titanium alloy in aerospace, due to its high strength-to-weight ratio, good corrosion resistance, and high thermal stability. However, due to its poor weldability, titanium sheets are preferably joined together by adhesive rather than riveting or welding. Surface modification of titanium by atmospheric pressure plasma treatment results increase in surface energy and, more interestingly, plasma-modified titanium surface is adequately stable prior to adhesive bonding. Therefore, single lap shear adhesive bond strength of plasma-treated titanium alloy is comparable to that of chemically modified titanium surface. ${ }^{16]}$

\section{Atmospheric pressure plasma treatment}

Polyimide sheets were plasma treated using TIGRES Plasma-BLASTER MEF equipment with operating voltage of $230 \mathrm{~V}$ and frequency of $60 \mathrm{~Hz}$. The treatment distance of polymer surface from nozzle head of plasma equipment was $5 \mathrm{~mm}$ and the gas used for treatment was air with a flow rate of $51 \mathrm{~L} /$ minute at a pressure of 4.5 bars.

Before performing the plasma treatment, the polymers were initially cleaned in methanol using ultrasonic method to remove any contamination on the surface. The specimens were then treated with atmospheric pressure plasma. In this investigation, the surface of the polymer was subjected to plasma treatment for 30 , 60 , and 120 seconds. The titanium specimens were washed with ethanol before joining with polyimide sheet to remove any contamination from surface.

\section{Contact angle measurement and surface energy estimation}

The surface energy for Polyimide Kapton sheet (Meldin 7001) was determined by using contact angle measurement. Contact angles were measured by sessile drop technique. Contact angle measurements were

Table I. Polar, Dispersion, and Total Surface Energy of Test Liquids

\begin{tabular}{lccc}
\hline Liquids & $\gamma_{\mathrm{LV}}^{\mathrm{P}}(\mathrm{m} \mathrm{N} / \mathrm{m})$ & $\gamma_{\mathrm{LV}}^{\mathrm{D}}(\mathrm{m} \mathrm{N} / \mathrm{m})$ & $\gamma_{\mathrm{LV}}(\mathrm{m} \mathrm{N} / \mathrm{m})$ \\
\hline Deionized Water & 50.2 & 22.0 & 72.2 \\
Formamide & 18.6 & 39.6 & 58.2 \\
\hline
\end{tabular}


performed by Modular CAM 200-Optical contact angle and surface tension meter using deionized water and formamide. The surface energy and its polar and dispersion components for Polyimide were calculated using the following equation ${ }^{[13]}$ :

$$
(1+\cos \theta) \gamma_{\mathrm{LV}}=2\left(\gamma_{\mathrm{S}}^{\mathrm{D}} \gamma_{\mathrm{LV}}^{\mathrm{D}}\right)^{1 / 2}+2\left(\gamma_{\mathrm{S}}^{\mathrm{P}} \gamma_{\mathrm{LV}}^{\mathrm{P}}\right)^{1 / 2}
$$

In Eq. [1], " $\theta$ " is the measured contact angle of liquid with the solid surface, $\gamma_{L V}$ is the total surface tension of the liquid, $\gamma_{\mathrm{LV}}^{\mathrm{D}}$ is the dispersion component of the liquid, and $\gamma_{\mathrm{LV}}^{\mathrm{P}}$ is the polar component of the liquid. The total surface tension' $\gamma_{\mathrm{LV}}$ ' of liquids and their polar ' $\gamma_{\mathrm{LV}}^{\mathrm{D}}$ ' and dispersion ' $\gamma \mathrm{Lv}$ ' components are known. Contact angles of both liquids on the solid surfaces were determined by the sessile drop method and the unknown dispersion and polar components of the Polyimide Kapton sheet surface were determined. Finally, the total surface energy ' $\gamma_{\mathrm{S}}$ ' was estimated by adding $\gamma_{\mathrm{S}}^{\mathrm{P}}$ and $\gamma_{\mathrm{S}}^{\mathrm{D}}$ as given in Eq. [2]:

$$
\gamma_{\mathrm{S}}=\gamma_{\mathrm{S}}^{\mathrm{D}}+\gamma_{\mathrm{S}}^{\mathrm{P}}
$$

\section{Adhesive joint preparation and lap shear tensile} testing

Rectangular specimens of Polyimide and Titanium sheets, with dimensions $100 \times 25 \times 3 \mathrm{~mm}^{3}$, were used for lap shear testing. The specimens were bonded for single lap shear tests. The tensile lap shear specimens were prepared by applying a high temperature-resistant Polyimide adhesive. Prior to the preparation of an adhesive joint, degassing of the adhesive was carried out under a pressure of $1 \mathrm{~Pa}$ for 10 minutes. Any excessive adhesive present at the interface was expelled by mechanical pressing of the joint, which resulted in a joint having an adhesive thickness of about $0.20 \mathrm{~mm}$. The adhesive thickness was controlled by adding $1 \mathrm{wt}$ pct of glass beads $(200 \mu \mathrm{m}$ diameter $)$ in the adhesive and applying a uniform pressure on all the adhesive-bonded joints. These samples were prepared according to ASTM standards D-1002 for single SLS testing. Pressure was applied to the lap joint during the curing cycle by two binder clips. The bonded specimens were cured at $448 \mathrm{~K}\left(175^{\circ} \mathrm{C}\right)$ for 1 hour with a subsequent post-curing at $483 \mathrm{~K}\left(210{ }^{\circ} \mathrm{C}\right)$ for 5 hours. The bonded specimens of PI and titanium were cured at $448 \mathrm{~K}\left(175^{\circ} \mathrm{C}\right)$ for 1 hour and subjected to subsequent post-cure at $483 \mathrm{~K}\left(210^{\circ} \mathrm{C}\right)$ for 5 hours. Four types of polyimide over lap joints were prepared and tested.

(a) Untreated Polyimide to polyimide joint

(b) Atmospheric plasma-treated polyimide joint with Polyimide

(c) Untreated Polyimide to titanium joint

(d) Atmospheric plasma-treated polyimide joint with titanium

Lap shear testing was carried out using a computercontrolled testing machine, ZWICK 2010, and a load cell of $50 \mathrm{kN}$. The specimens were loaded in tension at a test speed of $5 \mathrm{~mm} /$ minute. Five specimens were tested for each type of material combinations. All tests were performed at $295 \mathrm{~K}\left(22^{\circ} \mathrm{C}\right)$ with 50 pet humidity.

\section{SEM analysis of substrates surfaces}

Scanning electron microscopy was used to study the surface roughness of the Polyimide sheet before and after atmospheric pressure plasma treatment. Also a study on the fractured surface of the adhesive-bonded joint after lap shear test was carried out. Images were obtained using a JOEL JSM-7500F field emission scanning electron microscope (FE-SEM).

\section{Surface morphology investigation by AFM}

Applications of the state-of-the-art atomic force microscopy methods to elucidate the surface and nearsurface structure of polyimide sheet were performed. Atomic force microscopy (AFM) is used to examine topographical changes on the surface of polymers after plasma treatment. AFM produces topographical images which quantify surface morphology on an area scale, by scanning a sharp tip (typical end diameter 5 to $10 \mathrm{~nm}$ ) over the polymer surface.

AFM measurements were performed with a commercial AFM (NT-MDT Co Russia) in dynamic and phase contrast modes, and a cantilever (Nano Probe NCRC) having a spring constant tip $=48 \mathrm{~N} / \mathrm{m}$, resonance frequency $f_{\text {res }}=87 \mathrm{kHz}$, and a tip radius $r_{\text {tip }}<10 \mathrm{~nm}$ was applied. All measurements were carried out under controlled atmosphere $\left(T=295 \mathrm{~K} \quad\left(22^{\circ} \mathrm{C}\right)\right.$, relative humidity $R H=40 \mathrm{pct})$.

The roughness of PI sheet determined with AFM was analyzed using computer software. Two parameters such as the mean square roughness (RMS) and average roughness $(\mathrm{RA})^{[8]}$ are calculated. RMS represents the roughness for the square root of the arithmetic mean of the square of the absolute deviation from the average height, and $\mathrm{Ra}$ represents the roughness for the arithmetic mean of the absolute deviation from the average height.

\section{XPS analysis on unmodified and modified polyimide} surface

The XPS was carried out using an SSLSSX-100 X-ray photoelectron spectrometer by using monochromatized $\mathrm{AlK}_{\alpha}$ X-ray source, under operating power of $200 \mathrm{~W}$. The base pressure in the vacuum chamber during analysis was $2 \times 10^{-10} \mathrm{~Pa}$. Survey spectra were obtained using pass energy of $58.7 \mathrm{eV}$, and during the analysis, the electron take-off angle was set at $45 \mathrm{deg}$ and the acquisition time was 6 minutes.

\section{RESULTS}

\section{A. AFM Analysis of Surface Morphology of Polyimide Sheet}

AFM analysis of untreated polyimide sheet and 30, 60 , and 120 seconds atmospheric pressure plasmatreated sheets is shown in Figure 1.

Figure 1 indicates the surfaces of PI, modified by air plasmas not only incorporates cross-linking by activated 


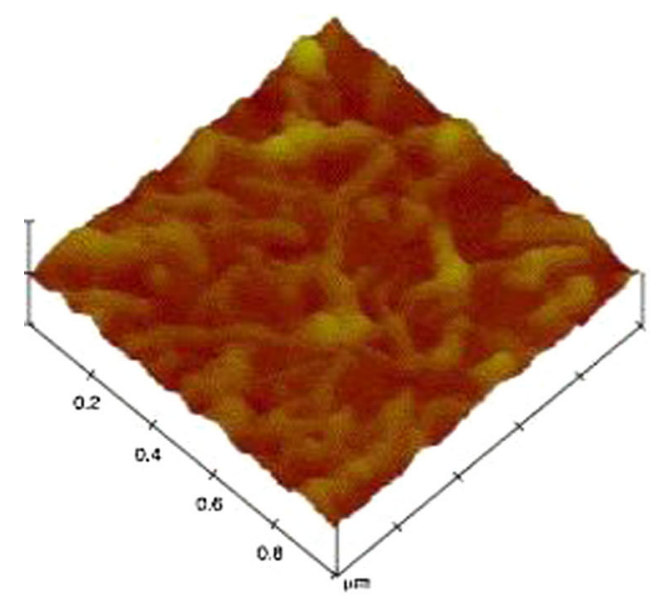

(a)

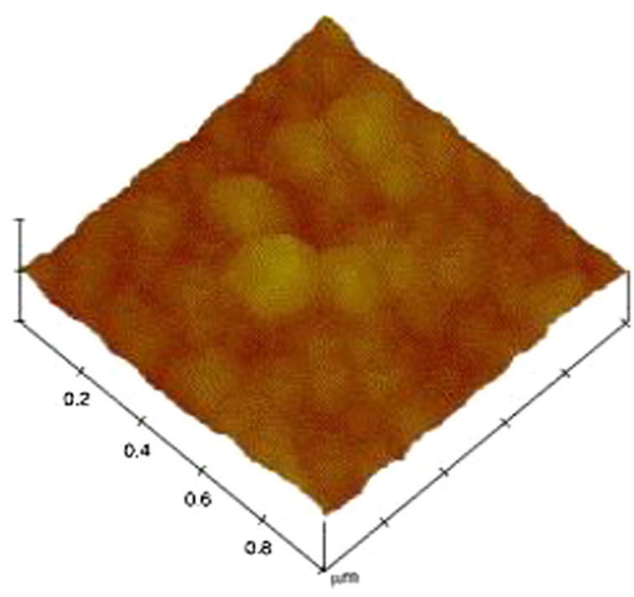

(c)



(b)

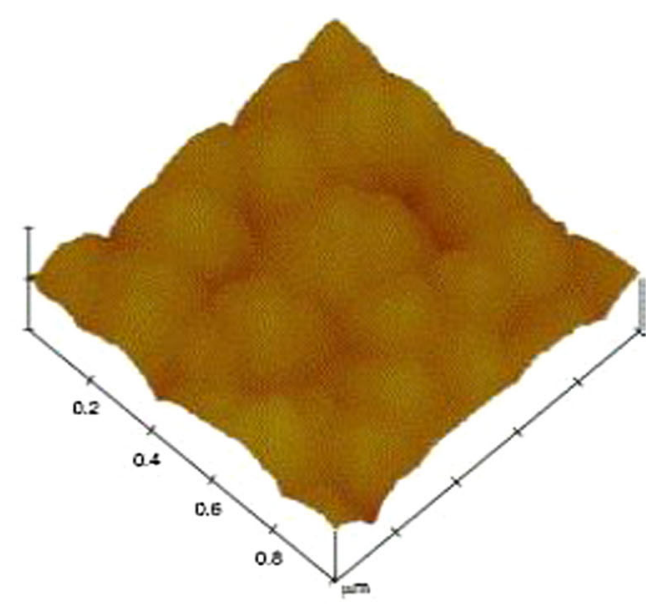

(d)

Fig. 1-The three-dimensional AFM pictures of (a) untreated, (b) $30 \mathrm{~s},(c) 60 \mathrm{~s}$, and $(d) 120 \mathrm{~s}$ of atmospheric pressure plasma-modified Kapton Sheet.

species of inert gases (CASING) of nitrogen radicals but also ablation by activated species of reactive gases (AASRG) of oxygen radicals. Apparently, air plasmas result in cross-linking on Polyimide Kapton surface. ${ }^{[17,18]}$

Figure 2 shows that in comparison to untreated PI, surface roughness of PI increases due to surface modification of PI under atmospheric pressure plasma. The optimal duration of time for air plasma modification was found to be 60 seconds. Figure 2 indicates that surface roughness increases considerably with increasing duration of plasma surface treatment.

\section{B. Surface Energy of Unmodified and Modified Polyimide Surface}

Surface energy and its polar and dispersion component after different plasma treatment time on Polyimide sheet are shown in Figure 3.

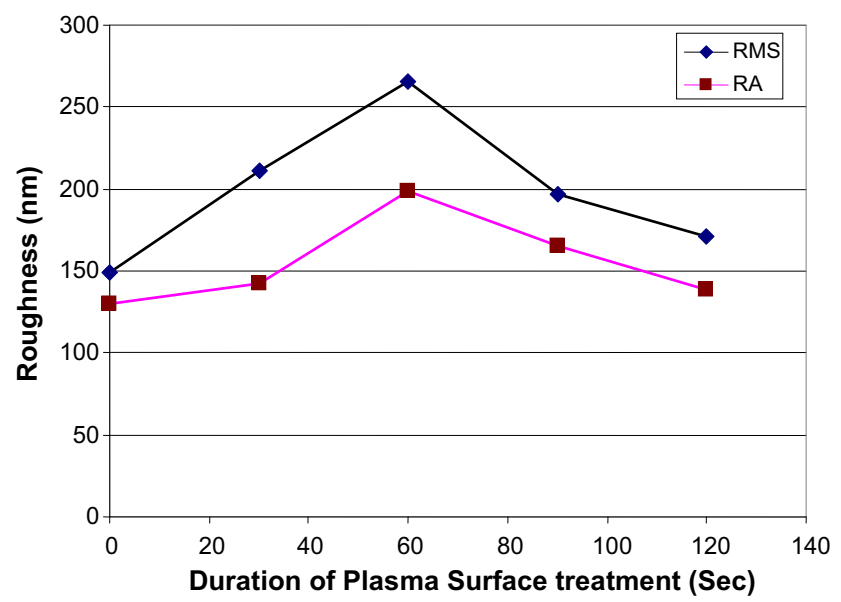

Fig. 2-The surface roughness "RMS" and "RA" of untreated and atmospheric plasma-treated PI with various time duration of plasma treatment on PI Sheet. 


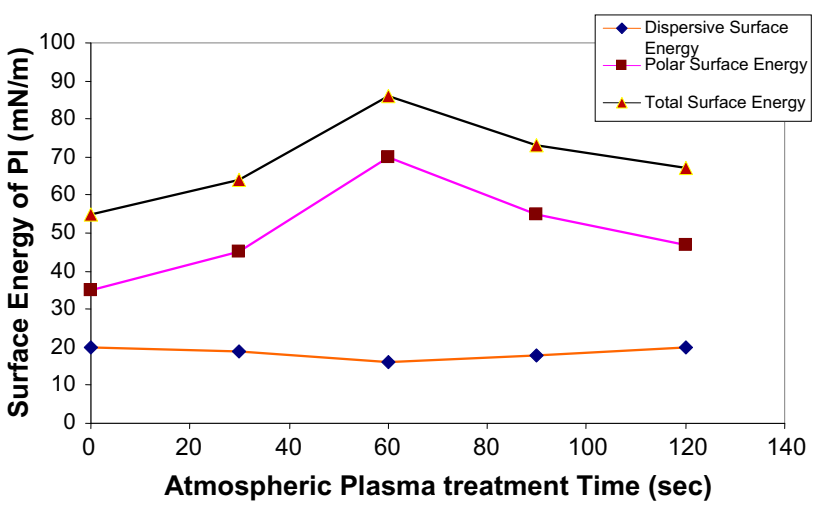

Fig. 3-The surface energy of unmodified and atmospheric plasmamodified polyimide sheet are plotted with various time durations for plasma modification on polyimide.

It is observed that due to surface modification of the polyimide sheet by atmospheric pressure plasma, there is a significant increase in surface energy of the polymer up to 60 seconds of exposure, in comparison to surface energy of the untreated polyimide. The surface energy of untreated polyimide is $54 \mathrm{mN} / \mathrm{m}$ and increases maximum up to $86 \mathrm{mN} / \mathrm{m}$. This figure shows that the dispersion component of surface energy of polyimide sheet after plasma treatment is decreased and polar component is increased. However, it is observed that the increase in polar component of surface energy is significantly higher when compared to corresponding decrease in dispersion component of surface energy. Therefore, a net gain in surface energy is achieved after plasma treatment. The increase in surface energy is 75 pct compared to that of the surface energy of polyimide before plasma treatment.

\section{XPS Studies on Unmodified and Modified Polyimide Surfaces}

XPS wide scan spectra of the unmodified polyimide surface shows $\mathrm{C}$ 1s peak, $\mathrm{O}$ 1s peak, and $\mathrm{N} 1 \mathrm{~s}$ as shown in Figure 4(a). However, due to the surface modification of polyimide under atmospheric pressure plasma, chemical functionalities of polyimide surface change as evident from Figure 4(b). In this case, the polyimide surface shows a significant increase in $\mathrm{O}$ 1s peak, and considerable decrease in $\mathrm{C} 1 \mathrm{~s}$ peak, as shown in Figure 4(b) and consequently oxygen-to-carbon $(\mathrm{O} / \mathrm{C})$ ratio increases considerably as shown in Figure 4(c).

The surface energy of untreated polyimide is $54 \mathrm{mN} /$ $\mathrm{m}$ and corresponding $\mathrm{O} / \mathrm{C}$ ratio is 0.04 and increases maximum up to $86 \mathrm{mN} / \mathrm{m}$ and corresponding $\mathrm{O} / \mathrm{C}$ ratio is 0.072. Apparently, due to incorporation of oxygen functionalities on the polyimide surface, the surface energy of the polymer increases resulting in increase in wetting characteristics of adhesive, thereby enhancing the adhesion characteristics of the polyimide surface.

\section{Lap Shear Tensile Properties of Adhesive-Bonded Joints}

Lap shear tensile strength tests were carried out on untreated and plasma-treated polyimide sheet bonded to

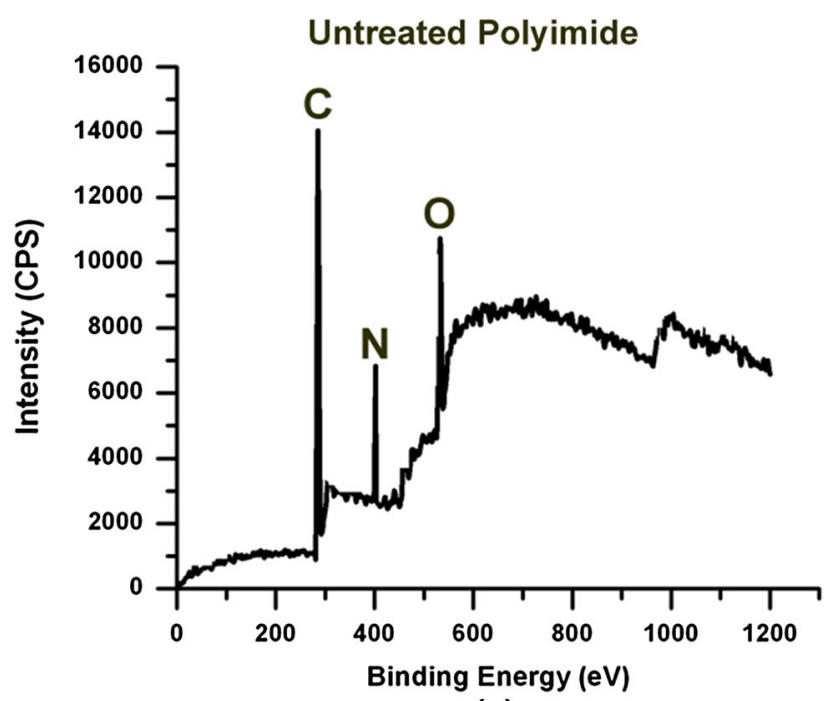

(a)

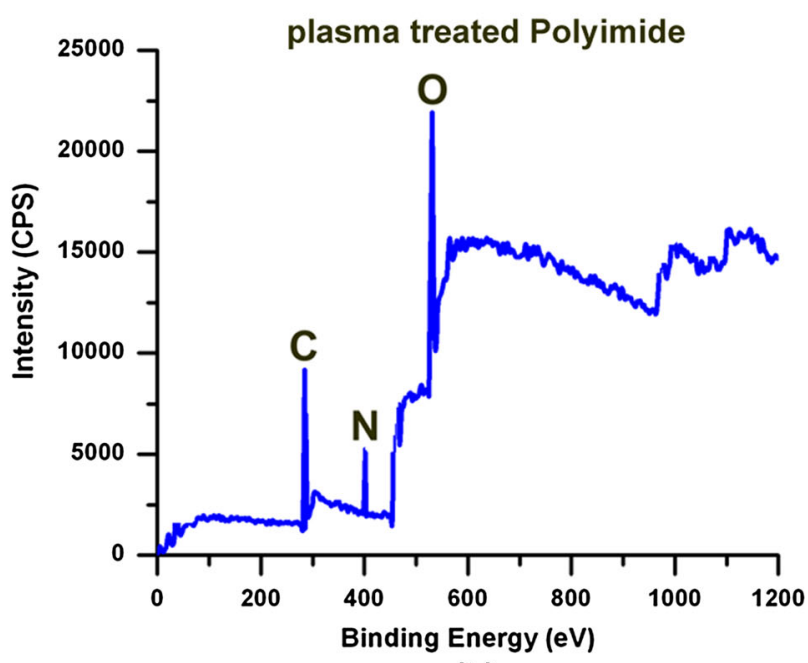

(b)

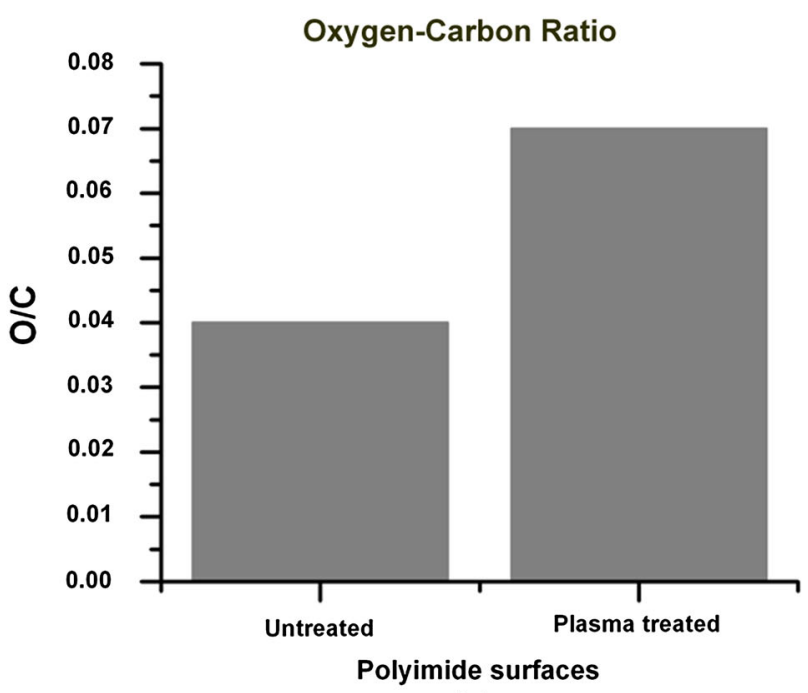

(c)

Fig. 4-XPS diagram of (a) unmodified polyimide, $(b)$ atmospheric plasma-modified polyimide, and $(c)$ oxygen/carbon ratio of unmodified and atmospheric plasma-modified polyimide. 


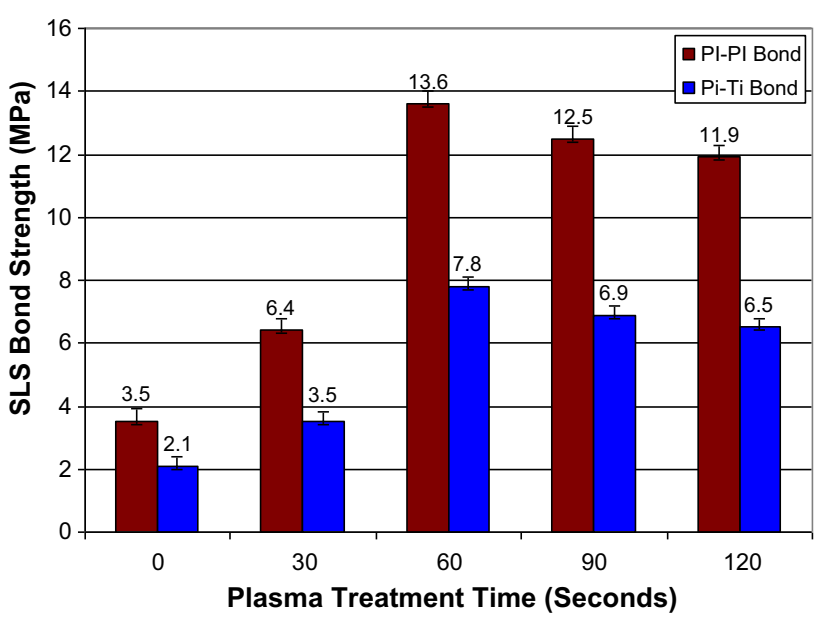

Fig. 5-Comparison of single lap shear adhesive bond strength untreated and plasma-treated polyimide sheet bonded with polyimide sheet and titanium sheet.

titanium and polyimide sheet bonded to polyimide sheet. A comparison of the experimental results is shown in Figure 5. The bond strength of atmospheric plasma-treated polyimide sheet with polyimide sheet increases with increase in plasma treatment time. The figure reveals that adhesive bond strength after $60 \mathrm{sec}-$ onds of treatment is almost increased two times than the adhesive bond strength after 30 seconds of plasma treatment and four times than the adhesive bond strength of untreated polyimide adhesive bond strength. Adhesive bond strength of untreated polyimide to polyimide is $3.5 \mathrm{MPa}$ and increases up to $13.6 \mathrm{MPa}$ due to atmospheric pressure plasma treatment.

Lap shear tensile strength of polyimide sheet bonded with titanium sheet after different durations of treatment time of atmospheric pressure plasma on polyimide surface is shown in Figure 5. It is evident from the figure that adhesive bond strength of polyimide to titanium also increases with plasma treatment but overall it shows lower adhesive bond strength in comparison to polyimide to polyimide adhesive bond strength. Adhesive bond strength of untreated polyimide to titanium is $2.1 \mathrm{MPa}$ and increases up to $7.8 \mathrm{MPa}$, when polyimide surface was modified by atmospheric pressure plasma treatment.

Figure 6 shows that lap shear tensile strength of polyimide to polyimide and polyimide to titanium is a function of $\mathrm{O} / \mathrm{C}$ ratio and it is interesting to note that with increasing $\mathrm{O} / \mathrm{C}$ ratio more precisely with increasing surface energy, adhesive bond strength increases.

Figure 7 shows a similar observation in terms of surface roughness of the polymer and the figure depicts that with increasing surface roughness of the polymer, adhesive bond strength polyimide to polyimide and polyimide to titanium increases.

\section{E. SEM Analysis of Surface Morphology of Polyimide Sheet}

Surface topography of polyimide sheet surface under scanning electron microscope, prior to and after atmo-

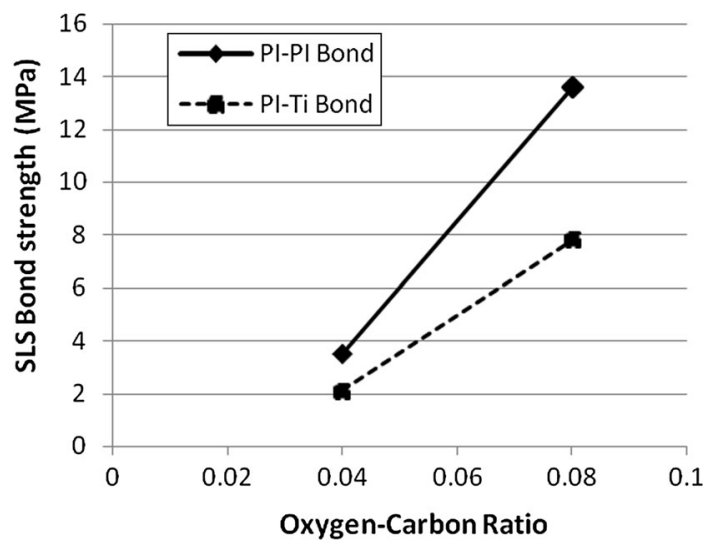

Fig. 6-Influence of oxygen-to-carbon ratio on polyimide on lap shear tensile strength of polyimide to polyimide and polyimide to titanium.

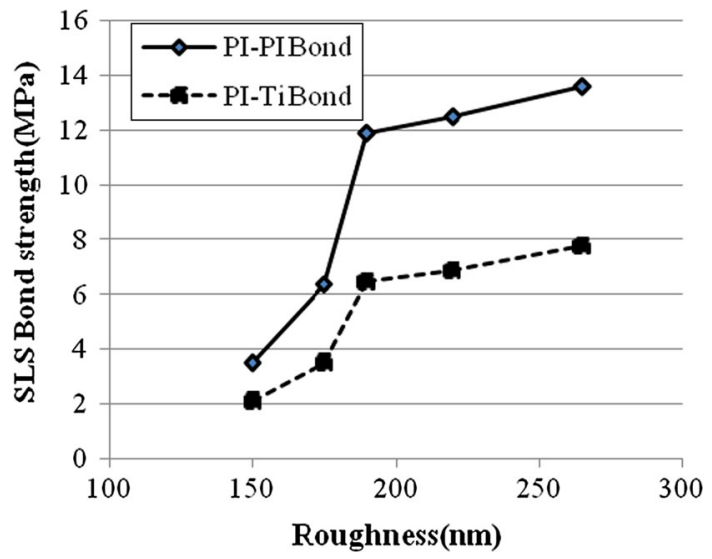

Fig. 7-Influence of surface roughness of polyimide on lap shear tensile strength of polyimide to polyimide and polyimide to titanium.

spheric plasma treatment of polyimide sheet, is shown in Figure 8.

It is evident from the figure that there is an increase in surface roughness of the polymer after plasma treatment. Therefore, it can be concluded that atmospheric pressure plasma treatment essentially helps in improving the adhesion properties of the polymer; consequently, failure is primarily cohesive from the adhesive resulting in higher adhesive bond strength.

\section{DISCUSSION}

This investigation highlights effect of atmospheric pressure plasma on adhesive bonding of polyimide to polyimide sheet and polyimide to titanium sheet. In this context, it is to be noted that atmospheric pressure plasma-treated polyimide to polyimide adhesive bonds show much stronger strength compared to atmospheric plasma-treated polyimide to titanium adhesive bond strength. This can be explained by the fact that polyimide-based adhesive shows similar chemical structure as that of polyimide sheet, and consequently, affinity to form stronger adhesive bond with polyimide 

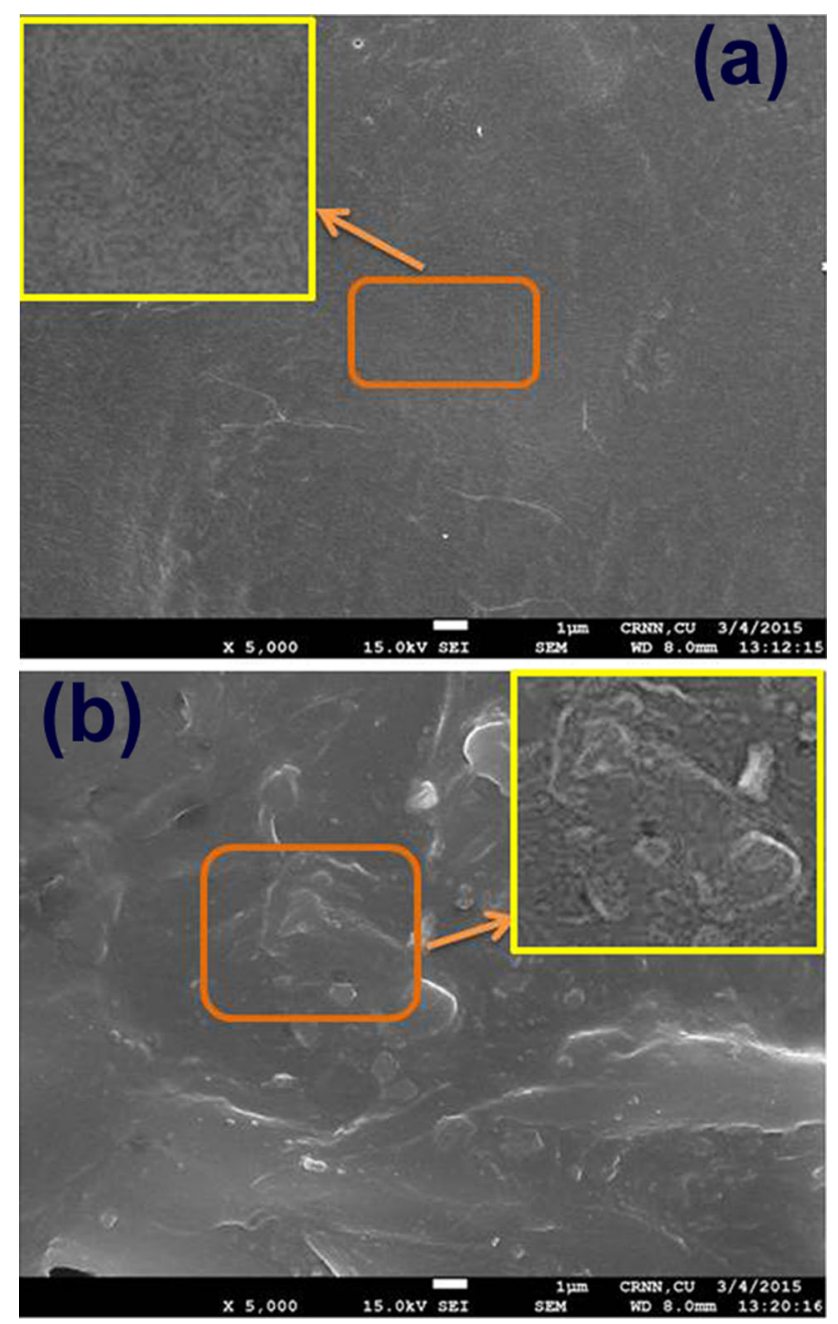

Fig. 8-SEM micrograph of polyimide surface before and after plasma treatment (a) untreated polyimide at 5000 times magnification and $(b)$ treated polyimide at 5000 times magnifications.

sheet increases. The mechanical stiffness of polyimide adhesive is closely comparable to polyimide sheet than that of titanium sheet resulting in more uniform distribution of interfacial stress. Therefore, in the case of polyimide to titanium adhesive bond testing, delamination may occur at adhesive-titanium interface rather than adhesive-polyimide interface.

The present investigation was also carried out to justify qualitatively and quantitatively the effect of atmospheric plasma treatment on the surface roughness and surface energy of polyimide leading to adhesion characteristics and failure mechanism of adhesivebonded joints. High-performance polymers very often do not possess the desired surface properties to form strong adhesive bonds. They are hydrophobic in nature and in general exhibit insufficient adhesive bond strength due to relatively low surface energy. ${ }^{[8,19]}$ Therefore, in order to improve the surface energy of polymers, the surfaces of Polyimide specimens were modified by atmospheric pressure plasma treatment. Surface energy analysis presented in Figure 3 suggests that surface energy of polyimide increases with duration of atmo- spheric pressure plasma modification up to 60 seconds and then decreases with increasing exposure time of plasma. The increase in surface energy of polyimide by atmospheric plasma treatment is mainly due to increase in polar component of surface energy. Therefore, it can be concluded that enhancement of adhesive bond strength by atmospheric pressure plasma treatment is a function of surface energy of polyimide.

Surface modification of polymer creates hydrophilic properties resulting in increase in adhesion. The improvement in adhesion properties is due to the formation of chemical bonds on the polymer surfaces. ${ }^{[14,20]}$ In addition, plasma treatment oxidizes the surface of the polymer to such an extent that adhesive interacts with oxidized layer resulting in strong adhesion. ${ }^{[10]}$ The present investigation also shows similar observation as evident from Figure 4. In certain cases, improvement of relative adhesive bond strength may increase ten to hundred times depending on substrate material and gas used for plasma. ${ }^{[21]}$ Surface modification with plasma treatment is confined to the top several hundred angstroms, and bulk properties of the material remain the same. ${ }^{[8,21]} \mathrm{X}$-ray Photoelectron Spectroscopy (XPS) analysis of Lin suggested that ${ }^{[22]}$ enhancement of adhesive bond strength is also dependent on relative composition of oxygen to carbon, which is similar to the observation of the present investigation as shown in Figure 4(c). Therefore, when plasma is applied on polymer surface, the surface of polymers not only induces cross-linking but also activated species of reactive gases (oxygen radicals) in atmospheric pressure plasma, demonstrated in Figures 1 and 4.

Polyimide kapton sheet shows different chemical bonds such as $\mathrm{C}-\mathrm{C} / \mathrm{C}-\mathrm{H}, \mathrm{C}-\mathrm{O}, \mathrm{C}=\mathrm{O}$, and $\mathrm{C}-\mathrm{N}$; consequently, when plasma is applied on polyimide surface from 0 to 60 seconds, it generates more free radicals on polyimide surface, which react with free oxygen and nitrogen in the air. Bowditch and Shaw have shown that when oxygen gas was used to modify the polymer surfaces, this results an increase in hydrophilicity of the polymer surface. ${ }^{[1]}$ It has been emphasized that the desired gas reacts with a wide range of polymers to produce various functional groups, including $\mathrm{C}-\mathrm{O}$, $\mathrm{C}=\mathrm{O}$, and $\mathrm{O}-\mathrm{C}-\mathrm{O}$ at the surface ${ }^{[8,10]}$ When exposure time of plasma increases further, the cross-linking of activated species of inert gases (CASING) on polymer surface reaches to a steady state and does not produce more free radicals on polymer surface. Therefore, ablation by activated species of reactive gas (oxygen) breaks more chemical bonds and thus leads to decreasing of surface composition of oxygen to carbon on polyimide film. ${ }^{[22-25]}$

Adhesion properties of polymer are also strongly influenced by the surface topography of the polymer. Noeske et al. and Thurston et al. have observed that the surface roughness of polymer samples increases after plasma treatment. ${ }^{[9,20]}$ The increase in surface roughness attributes an increase in adhesion of polymers. In this context, the present investigation also supports this statement as AFM analysis and SEM microscopic studies on the polymeric surface after atmospheric pressure plasma treatment clearly show an increase in 
surface roughness as evident from Figure 1. Therefore, it can be concluded from Figures 2 and 3 that increase in adhesive bond strength is a function of surface roughness of RMS for polyimide.

The microscopic roughness can also be attributed to better mechanical interlocking leading to increase in adhesive bond strength. However, the most important contribution of atmospheric pressure plasma treatment on the polyimide sheet is the significant increase in surface energy of the polyimide. The polar component of surface energy of the polymers are primarily responsible for the increase in surface energy of the polymer due to the formation of the polar functional groups on the surface of the polymers, leading to better adhesion characteristics. $^{[20]}$ The improvement in adhesive bond strength with atmospheric pressure plasma treatment could be explained with the fact that atmospheric pressure plasma treatment essentially improves hydrophilic properties of polymer. Further, surface morphology of polymer improves in terms of adhesion by increasing the surface roughness. ${ }^{[21,23]}$ Therefore, atmospheric pressure plasma treatment on polymer surface is beneficial in two ways, first, by increasing the surface energy by incorporating different functional groups and second by increasing the surface roughness which ultimately provides the effect of mechanical interlocking. ${ }^{[26]}$

It is observed that adhesive-bonded joints fail with a variety of failure modes. After atmospheric pressure plasma treatment, the locus of failure transferred from the adhesive-substrate interface to within adhesive. ${ }^{[26]}$ Figure 5 shows that adhesive bond strength of polyimide to polyimide shows higher adhesive bond strength in the case of untreated as well as atmospheric pressure plasma-treated polyimide in comparison to adhesive bond strength of polyimide to titanium. It could be explained that in the case of polymer and metal adhesive bond, the two dissimilar materials show different stiffness under tensile load, and consequently, exhibit low adhesive bond strength due to non-uniform distribution of stress across the interface.

Therefore, the present investigation concludes with a high note that application of atmospheric pressure plasma treatment is a very effective method for promoting strong adhesion properties within similar materials as well as dissimilar materials, with more precisely for high-performance adhesive-bonded joints.

\section{CONCLUSIONS}

The present investigation concludes that surface energy of polyimide increases significantly due to atmospheric pressure plasma treatment. Adhesive bond strength is considerably dependent on surface roughness and surface energy of the polyimide surface. Formation of chemical functional groups, and increase in surface roughness and surface energy after plasma treatment improve adhesion properties of polyimide. Due to atmospheric pressure plasma treatment on polyimide, adhesive bond strength increases significantly. Adhesive bonding of similar materials shows higher adhesive bond strength in comparison to dissimilar materials.

\section{REFERENCES}

1. M.R. Bowditch and S.J. Shaw: J. Adv. Perform. Mater., 1996, vol. 3 (3-4), pp. 325-42.

2. J.M. Shenton, M.C. Lovell-Hoare, and G.C. Stevens: $J$ Phys. D, 2001, vol. 34 (18), pp. 2754-60.

3. A.M.R.G. Urena and G. Prolongo: 13th European Conference on Composite Materials 2008.

4. J. Custodio, J.G. Broughton, H. Cruz, and P. Winfield: Int. J. Adhes. Adhes., 2009, vol. 29 (2), pp. 167-72.

5. J. Shields: Adhesive Bonding, Oxford University Press, London, 1974.

6. W. Leahy, W. Barron, M. Buggy, T. Young, A. Mas, F. Schue, T. McCabe, and M. Bridge: J. Adhes., 2001, vol. 77 (3), pp. 215-49.

7. S. Bhowmik, H.W. Bonin, V.T. Bui, and R.D. Weir: J. Appl. Polym. Sci., 2006, vol. 102 (2), pp. 1959-67.

8. C.M. Chan, T.M. Ko, and H. Hiraoka: Surf Sci Rep., 1996, vol. 24 (1-2), pp. 1-54.

9. M. Noeske, J. Degenhardt, S. Strudhoff, and U. Lommattzsch: Int. J. Adhes. Adhes., 2004, vol. 24 (2), pp. 171-77.

10. E.M. Petrie: Hand Book of Adhesives and Sealants, 2nd ed., McGraw Hill, New York, 2007.

11. J.I. Rotheiser and J. Rotheiser: Joining of Plastics, 3rd ed., Hanser Gardner Publications, Cincinnati, OH, 2009.

12. F.D. Egitto and L.J. Matienzo: IBM J. Res. Dev., 1994, vol. 38 (4), pp. 423-39.

13. J. Comyn: Int. J. Adhes. Adhes., 1992, vol. 12 (3), pp. 145-49.

14. J. Custodio, J. Broughton, and H. Cruz: Int. J. Adhes. Adhes., 2009, vol. 29 (2), pp. 173-85.

15. J. Eisinger: Basic Principles in Nucleic Acid Chemistry, Academic Press, New York, 1974, pp. 399-452.

16. M. Akram, K.M.B. Jansen, L.J. Ernst, and S. Bhowmik: Int. J. Adhes. Adhes., 2011, vol. 31 (7), pp. 598-604.

17. F.D.M. Egitto: IBM J. Res. Dev., 1994, vol. 38 (4), pp. 423-39.

18. H. Yasuda: in Plasma Polymerization, Chapter 10, H. Yasuda, ed., Academic Press, New York, 1985, pp. 334-69.

19. S.Y. Xu, S. Guo, and D.A. Dillard: J. Adhesion., 2004, vol. 80 (12), pp. $1153-72$

20. R.M. Thurston, J.D. Clay, and M.D. Schulte: J. Plast. Film Sheeting., 2007, vol. 23 (1), pp. 63-78.

21. S. Han, Y. Lee, H. Kim, G. Kim, J. Lee, J. Yoon, and G. Kim: $J$. Surf. Coat. Technol., 1997, vol. 93 (2-3), pp. 261-64.

22. Y.S. Lin, H.M. Liu, and C.L. Chen: J. Surf. Coat. Technol., 2006, vol. 200 (12-13), pp. 3775-85.

23. Y. Kim, Y. Lee, S. Han, and K.-J. Kim: J. Surf. Coat. Technol., 2006, vol. 200 (16-17), pp. 4763-69.

24. C. Mandolfino, E. Lertora, and C. Gambaro: J. Adv. Aerosp. Eng., 2014, vol. 2014, pp. 1-7.

25. X. Dong, C. Ritts, C. Staller, Q. Yu, M. Chen, and Y. Wang: Eur. J. Oral Sci., 2013, vol. 121 (4), pp. 355-62.

26. J. Schaferabc, T. Hofmanna, J. Holtmannspottera, M. Frauenhoferb, J. vonCzarneckia, H.J. Gudlad: J. Adhes. Sci. Technol., 2015, Doi: 10.1080/01694243.2015.1037380. 